\title{
Refine penetrance estimates in the main pathogenic variants of Transthyretin Familial Amyloid Polyneuropathy (TTR-FAP) by use of a new non-parametric approach (NPSE)
}

Farida Gorram ${ }^{1,2,3}$, Flora Alarcon ${ }^{4}$, Hervé Perdry ${ }^{5}$, Bérénice Hébrard ${ }^{6}$, Thibaud Damy ${ }^{2,3,7}$, Pascale Fanen ${ }^{3,}$ ${ }^{6}$, Benoît Funalot ${ }^{3,6,8}$, Gregory Nuel ${ }^{9}$ and Violaine Planté-Bordeneuve ${ }^{1,2,3}$

${ }^{1}$ Department of Neurology, Henri Mondor University Hospital, AP-HP, Créteil, France. ${ }^{2}$ Amyloid research Institute, Amyloid Network, Créteil, France. ${ }^{3}$ Paris-Est Créteil University, Créteil, France. ${ }^{4}$ Laboratory MAP5 UMR CNRS 8145 Paris Descartes University, France. ${ }^{5}$ University Paris-Sud, UMR-S 669 - Inserm, U669, Villejuif, France. ${ }^{6}$ Department of genetics, Henri Mondor University Hospital, AP-HP, Créteil, France. ${ }^{7}$ Department of Cardiology, Henri Mondor University Hospital, AP-HP, Créteil, France. ${ }^{8}$ Inserm U.955, Institut Mondor de Recherche Biomédicale, Créteil, France. ${ }^{9}$ Institute of Mathematics, National Center for French Research, Laboratory of Probability, University Pierre et Marie Curie, Sorbonne University, France.

Address for correspondence: Violaine Planté-Bordeneuve of Department of Neurology, Henri Mondor University Hospital, AP-HP, Créteil, France. E-mail: Violaine.plante@aphp.fr

Background: Significant variability of phenotype and age of onset are well known in TTR-FAP associated to a wide spectrum of pathogenic TTR variants, among which Val30Met is the most frequent $[1,2]$. Recently, new therapeutic options became available in TTR-FAP that should be administered from the very onset of symptoms. In this context, the knowledge of the risk of being symptomatic for mutation carriers (penetrance) is essential to adjust the follow-up of carriers and for patient management $[3,4]$. This study aims to refine penetrance estimates in the main pathogenic TTR variants encountered in our TTR-FAP population using a newly developed non parametric approach named NPSE for Non-Parametric Survival Estimate.

Materials and methods: A systematic genealogical enquiry was carried out in each family assessed in our center. Relevant data were collected with special attention to the genotype, phenotype, age at onset ( $\mathrm{AO}$ ) for affected individuals and age at last news for asymptomatic carriers and relatives. Portuguese families were not included in this study.

In previous works, we estimated penetrance as function of age, using a parametric survival method the PEL (Proband's Exclusion Likelihood) [6] in which the AO is modeled by a Weibull distribution (WD). However, such method does not fit properly when penetrance function is far from WD. To avoid this bias, we have developed a non-parametric approach NPSE [5] based on a survival analysis (Kaplan-Meier estimator) that fits any penetrance shape. Also, NPSE can take into account covariates, here the TTR variant, through a Cox Model.

Results: We obtained genealogical data from 71 unrelated kindreds, including Val30Met (35 families, 90 patients), Ser77Tyr (15 families, 47 patients), Ile107Val (12 families, 21 patients), Ser77Phe (9 families, 30 patients) with information on 1654 subjects (188 affected /115 asymptomatic carriers).

Mean age at onset $( \pm$ Standard Deviation ) was 54.6 years $( \pm 14.9)$ for Val30Met, 55.6 years $( \pm 9.1)$ for Ser77Tyr, 58.7 years $( \pm 6.4)$ for Ser77Phe and 62.3 years $( \pm 6.8)$ for Ile107Val.

Penetrance estimates were significantly different between the 4 TTR variants tested $(p=0.003)$ and remained incomplete in elderly. Hence, at 80 years of age, penetrance (95\% Confidence Interval) varies from 46.9\%, (16.6-66.2) in Ile107Val up to 71.3\% (51.1-83.1) for Ser77Tyr kindred.

The disease risk is virtually null up to the age of 50 years for all variants except in the Val30Met group where it increases progressively from $3.1 \%$ (95\% CI, 0.3-5.7) at 30 years to $16.5 \%(9.8-22.7)$ at 50 and $64.4 \%(49.4-74.9)$ at 80 years old. In contrast, in non Val30Met variants, the risk increased abruptly after 50 years old. In Ser77Phe and Ser77Tyr carriers, it raised from 7\% (0.8- 
15.8 ) and $12.8 \%$ (3.3-21.4) respectively up to $65.8 \%$ (37.1-81.4) and $71.3 \%$ (51.1-83.1). Risk estimate was found the lowest in Ile107Val families where it increases progressively from 50 years to $37.5 \%(14.2-54.4)$ at $70 \mathrm{y}-0$.

Discussion and Conclusions: This study showed significant differences of penetrance profiles in TTR-FAP with various pathogenic mutations of TTR. Our results should be replicated and implemented on larger samples of families to refine the management of carriers. NPSE method is more accurate and powerful than PEL previously used to unravel penetrance estimates. In future studies, it will allow testing the effect of other covariates, like gender, origin, and parent of origin on disease expression.

Declaration of interest: The authors declare no conflict of interest.

References: 1. Plante-Bordeneuve V, Carayol J, Ferreira A, Adams D, Clerget-Darpoux F, Misrahi M, Said G, et al. Genetic study of transthyretin amyloid neuropathies: carrier risks among French and Portuguese families. J Med Genet 2003; 40:e120. 2. Plante-Bordeneuve V, Said G. Familial amyloid polyneuropathy. The Lancet Neurology 2011; 10(12):1086\{1097. 3. Hellman U, Alarcon F, Lundgren HE, Suhr OB, Bonaiti-Pellie C, Plante-Bordeneuve V. Heterogeneity of penetrance in familial amyloid polyneuropathy, ATTR Val30Met, in the Swedish population. Amyloid: J Protein Fold Dis 2008;15:181- 186. 4. Saporta MA, Zaros C, Cruz MW, André C, Misrahi M, BonaïtiPellié C, et al. Penetrance estimation of TTR familial amyloid polyneuropathy (type I) in Brazilian families. Eur J Neurol 2009; 16:337-41. 5. Alarcon F, Nuel G, Plante-Bordeneuve V (http://arxiv.org/abs/1607.04215). 6. Alarcon F, Bourgain C, Gauthier-Villars M, PlanteBordeneuve V, Stoppa-Lyonnet D, Bonaiti-Pellie C. PEL: an unbiased method for estimating age dependent genetic disease risk from pedigree data unselected for family history. Genet Epidemiol $2009 ; 33: 379-385$. 